DOI: 10.12957/demetra.2015.15555

\title{
Elaboração de aplicativo de avaliação nutricional para telefones celulares com sistema Android
}

Nutritional assessment application for mobile phones with Android system

Daniele Lima da Cruz'

Márcia Nacif ${ }^{1}$

${ }^{1}$ Universidade Presbiteriana Mackenzie, Curso de Nutrição, Centro de Ciências Biológicas e da Saúde. São Paulo-SP, Brasil.

Subvencionado pela Universidade Presbiteriana Mackenzie, por meio de bolsa de iniciação científica.

Correspondência / Correspondence

Daniele Lima da Cruz

E-mail: danny_Ic@msn.com

\section{Resumo}

Novas ferramentas de orientação nutricional vêm sendo utilizadas por diversos profissionais de saúde. Dentre estas, encontram-se os aplicativos de celular voltados à saúde que possuem diversos recursos e podem auxiliar o usuário a manter uma alimentação saudável. Nesse contexto, o estudo teve por objetivo elaborar um aplicativo de avaliação nutricional para telefones celulares com sistema Android. Foi desenvolvido um aplicativo denominado Diet Help, composto por sete funcionalidades: "Perfil do usuário", "Registro Alimentar, "Resumo do dia, "Histórico da dieta", "Calorias dos alimentos", "Alimentação saudável" e "Excluir usuário". As principais linguagens de programação utilizadas foram Android Java, Javascript e SQlite para banco de dados. O Diet Help permite cálculo do índice de massa corporal, classificação do estado nutricional de acordo com idade e sexo, informa necessidade energética diária recomendada, quantidade de calorias e nutrientes consumidas em cada refeição e se o consumo está de acordo com o recomendado para a idade e sexo do usuário. Também permite a consulta da quantidade de calorias e nutrientes por $100 \mathrm{~g}$ de qualquer alimento e possui informativo sobre alimentação saudável, com base no Guia Alimentar para a População Brasileira.

Palavra-chave: Avaliação Nutricional. Paciente. Dieta. Telefones Celulares. 


\section{Abstract}

New tools of nutritional orientation have been used by various health professionals. Among these, are the cell phone applications focused on health with several resources that can help the user to maintain a healthy diet. In this context, this study had as main objective to elaborate a nutritional assessment application for mobile phones with Android system. A nutritional assessment called Diet Help was developed, with seven screens: "User Profile", "Food Record", "Summary of the day", "Diet's history", "Food calories", "Eating healthy" and "Delete User". The main programming languages used were Android Java, Javascript and SQlite for database. The Diet Help allows calculation of body mass index, nutritional status according to age and sex, it informs recommended daily energy need, amount of calories and nutrients consumed at every meal and if this use is in accordance with the recommended for their age and user sex, allowing you to see the amount of calories and nutrients per $100 \mathrm{~g}$ of any food; it has information on healthy eating based on the Food Guide for the Brazilian Population [Guia Alimentar para a População Brasileira].

Key words: Nutritional Assessment. Patient. Diet. Cell Phones.

\section{Introdução}

Para uma vida saudável, são indispensáveis uma alimentação e nutrição adequadas, além da prática regular de atividade física. ${ }^{1} \mathrm{O}$ padrão alimentar de um indivíduo pode definir seu estado de saúde, crescimento e desenvolvimento ao longo da vida. ${ }^{2}$ Adotar hábitos alimentares saudáveis de acordo com parâmetros nacionais e internacionais de nutrição pode diminuir o risco de doenças, como as doenças crônicas não transmissíveis (DCNTs), que incluem alguns tipos de câncer, diabetes e doenças cardiovasculares. ${ }^{3}$

Os progressos científicos e tecnológicos ocorridos nas últimas décadas tornaram os alimentos mais disponíveis e facilitaram seu consumo, assim como permitiram que tarefas desgastantes e demoradas pudessem ser realizadas em um curto período de tempo e com gasto energético mínimo, ocasionando ganho de tempo e dinheiro. A princípio, tais mudanças deveriam melhorar o estado nutricional da população mundial, contribuindo para sua longevidade e qualidade de vida. O que se observa, no entanto, é um aumento no consumo de gorduras (principalmente a gordura saturada) e sódio, em detrimento do consumo de frutas e hortaliças, além do aumento da inatividade física. ${ }^{4}$ 
A modernidade levou as pessoas a buscarem a diversidade, representada por novos produtos, serviços e locais de alimentação, o que está ligado ao desejo de simplificar o momento da refeição. São exemplos, os produtos industrializados e os restaurantes do tipo self-service. ${ }^{5}$ A alimentação fora de casa também é um fator que contribui para o aumento da prevalência de DCNTs, pois é em sua maioria menos saudável, com maior densidade calórica, quantidade de açúcares, sal e gorduras, pobre em fibras e rica em sódio, quando comparada aos alimentos preparados em domicílio. ${ }^{6}$

Para auxiliar os indivíduos a expandir sua consciência sobre escolhas adequadas e prevenir o desenvolvimento de DCNTs, novas ferramentas, como a internet, vêm sendo utilizadas por diversos profissionais de saúde para veicular informações sobre alimentação saudável e prevenção de doenças. Dentre as novas ferramentas, encontram-se os aparelhos de telefone celular, que reúnem inúmeros recursos cujo foco há muito tempo deixou de ser apenas efetuar e receber chamadas. ${ }^{7}$ Os recursos presentes nos aparelhos móveis atuais são ágeis e fáceis de usar, proporcionando facilidade no acesso às informações e melhor suporte para aplicativos multimídia. ${ }^{8}$ Grande parte dos aplicativos pode ser adquirida gratuitamente, o que torna possível a experimentação de vários produtos. Além disso, aplicativos voltados à saúde estão entre os mais populares. ${ }^{9}$

Dentro desse contexto, este estudo teve como principal objetivo elaborar um aplicativo de avaliação nutricional para telefones celulares com sistema Android.

\section{Metodologia}

Trata-se de estudo no qual foi desenvolvido um aplicativo de avaliação nutricional para ser "baixado" e instalado em telefones celulares com sistema Android. Para seu desenvolvimento, foram utilizadas as seguintes linguagens:

- Android Java: linguagem padrão para desenvolvimento de aplicativos para plataforma Android. Utilizada para o carregamento inicial do software.

- Apache Cordova: conjunto de APIs (no português, interface de programação de aplicativos) que permite que um aplicativo para Android "rode" de forma nativa e ainda assim seja desenvolvido apenas utilizando HTML, CSS e Javascript.

- JavaScript e JQuery Mobile: utiliza Javascript (através da biblioteca JQuery Mobile) para implementar as funções do sistema, bem como a interação com o usuário.

- HTML e CSS: HTML é uma linguagem de marcação utilizada para criar a interface gráfica. Com ele, define-se o conteúdo a ser apresentado e com o CSS determina-se a aparência deste conteúdo.

- SQLite: banco de dados utilizado em aplicativos móveis. 
O aplicativo é constituído por uma tela inicial, em que o usuário tem seis opções:

Primeira opção: permite o preenchimento do Perfil dos Usuários com os dados pessoais para sua caracterização: sexo (feminino/masculino) data de nascimento (dd/mm/aaaa) e nível de atividade física (sedentário, pouco ativo, ativo e muito ativo). Também é solicitado que o usuário preencha os dados de peso $(\mathrm{kg})$ e altura $(\mathrm{m})$ para cálculo do índice de massa corporal (IMC) por meio da fórmula IMC $(\mathrm{Kg} / \mathrm{m} 2)=\mathrm{P} / \mathrm{A}^{2}$. O IMC é classificado de acordo com as curvas de crescimento do Ministério da Saúde se a idade do usuário estiver entre 10 e 18 anos. ${ }^{10}$ Se a idade do usuário estiver entre 19 e 60 anos de idade, a classificação é feita conforme a World Health Organization, ${ }^{11}$ e se a idade do usuário for maior que 60 anos, classifica-se o indivíduo de acordo com a Organização Pan-Americana de Saúde. ${ }^{12}$

No primeiro acesso ao sistema, o preenchimento do perfil é obrigatório. Alterações posteriores ficarão a critério do usuário. Nesta mesma tela, o usuário pode editar os dados informados. E ainda há a opção de cadastrar um novo perfil caso haja um segundo indivíduo utilizando o mesmo aparelho celular. Outros perfis possuem as mesmas opções que o usuário principal.

Todas as vezes que um novo perfil começar a ser preenchido, o usuário receberá o seguinte aviso: "As informações e sugestões contidas neste aplicativo têm caráter meramente informativo e não substituem o aconselhamento e o acompanhamento de um nutricionista".

A partir dos dados de idade, sexo, peso, altura e nível de atividade física (AF), calcula-se o Requerimento Energético Estimado (Estimated Energy Requirement-EER) pelas fórmulas estipuladas pela Dietary Reference Intake (DRIs). ${ }^{13}$ Se o paciente está na faixa de eutrofia, o EER é calculado com o peso informado. Porém, se o usuário está na faixa de desnutrição e sobrepeso, é calculado o peso ideal por meio da fórmula: Peso ideal $=21,7 / \mathrm{A}^{2}$. Se o usuário está na faixa de obesidade grau I, é calculado o peso ideal pela fórmula: peso ideal $=27,4 / \mathrm{A}^{2}$. Caso o usuário esteja na faixa de obesidade grau II, o peso ideal é feito através da fórmula: peso ideal = 32,4/A². E se o usuário está na faixa de obesidade mórbida, é calculado o peso ideal através da fórmula: peso ideal = 54,9/A².

A distribuição dos macronutrientes é feita segundo as DRIs, sendo de 45 a $65 \%$ do valor calórico total da dieta (VCT) de carboidratos, proteínas (10 a 35\%) e lipídeos (20 a 35\%). Estes dados são mostrados ao usuário no ícone abaixo do valor de EER.

Segunda opção: é o preenchimento de um Registro Alimentar. O indivíduo deve informar a refeição consumida (café da manhã, lanche da manhã, almoço, lanche da tarde, jantar e ceia), qual o alimento ingerido e sua respectiva quantidade (gramas ou medida caseira). À medida que o usuário cadastra um novo alimento, o sistema informa a quantidade de calorias consumidas até aquele momento. Para o cálculo das calorias e demais nutrientes consumidos, o aplicativo utiliza a Tabela de Composição Nutricional dos Alimentos Consumidos no Brasil - Tabela IBGE, ${ }^{14}$ assim 
como para referência das medidas caseiras. A quantidade de calorias é calculada por meio da seguinte fórmula: $\mathrm{Kcal}=$ carboidratos $(\mathrm{g}) * 4+$ proteínas $(\mathrm{g}) * 4+$ lipídeos $(\mathrm{g}) * 9$.

Terceira opção: é o Resumo do Dia, em que o usuário tem acesso a todas as informações referentes à alimentação consumida, incluindo a quantidade de calorias, a porcentagem de calorias que correspondem a carboidratos, proteínas e lipídios, quantidade de colesterol, fibra alimentar, vitaminas e minerais. O aplicativo avalia as vitaminas lipossolúveis (A, D, E e K), vitaminas hidrossolúveis (tiamina, riboflavina, piridoxina e niacina), vitamina C e minerais (cálcio, ferro, sódio e potássio).

Quarta opção: constituído pelo Histórico da Dieta. Permite ao usuário ter acesso a todos os dados referentes aos alimentos consumidos até aquele momento, separados por data. Nesta opção, o usuário consegue identificar se o consumo de nutrientes está acima ou abaixo do recomendado pelas DRIs. Caso o consumo esteja de acordo com o recomendado, os dados são informados na cor verde; caso estejam inadequados, aparecem em vermelho.

Quinta opção: permite a consulta de calorias (Calorias dos Alimentos) de qualquer alimento segundo a Tabela IBGE. ${ }^{14}$ Informa-se também a quantidade de proteínas, carboidratos, lipídios, colesterol, fibra alimentar, vitaminas lipossolúveis (A, D, E e K), vitaminas hidrossolúveis (tiamina, riboflavina, piridoxina e niacina), vitamina C e minerais (cálcio, ferro, sódio e potássio) por 100 gramas do alimento.

Sexta opção: permite acesso à tela Alimentação Saudável, em que o usuário tem informações acerca dos objetivos do programa, funcionalidades e dicas sobre alimentação saudável, com base no Guia Alimentar para a População Brasileira, incluindo "Os dez passos para uma alimentação saudável" presentes no mesmo documento. ${ }^{15}$

Quando o sistema fica inativo por 48 horas, ou seja, o usuário deixa de preencher seu registro alimentar por esse período, ele recebe o seguinte aviso: "Você não tem me visitado. Não deixe de cadastrar sua dieta”.

Todos os dados dos indivíduos são enviados via internet e armazenados em um servidor, onde cada usuário recebe um código de identificação para que sua identidade seja preservada.

\section{Resultados e Discussão}

Foi elaborado com sucesso um aplicativo de avaliação nutricional para telefones celulares com sistema Android, que foi nomeado Diet Help.

Para seu desenvolvimento, foram utilizadas as linguagens de programação Android Java, Apache Cordov, Java Script, JQuery Mobile, HTML, CSS e SQLite. A maior parte das linguagens usadas neste aplicativo também foi utilizada por Fernandes et al., ${ }^{16}$ ao desenvolverem um software Web e 
dispositivos móveis que auxiliassem o usuário a ter uma alimentação saudável, aliada à prática de exercícios físicos.

O aplicativo oferece sete opções de funcionalidades ao usuário, conforme a figura 1.

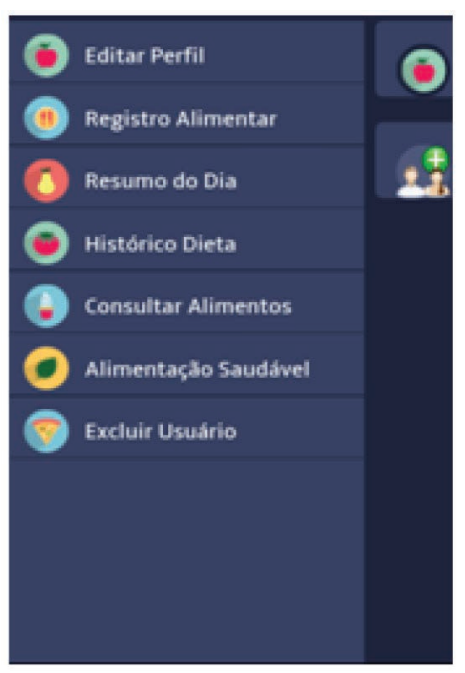

Figura 1. Menu principal do aplicativo. São Paulo, 2014.

Na primeira tela do aplicativo, é feito o cadastro de um novo usuário, onde primeiramente surge o aviso: "As informações e sugestões contidas neste aplicativo têm caráter meramente informativo e não substituem o aconselhamento e o acompanhamento de um nutricionista." Tal informação foi inserida no aplicativo, pois de acordo com o Código de Ética do Nutricionista, Capítulo IV da responsabilidade profissional, artigo 6⿳⺈, parágrafo XVII: "É vetado ao nutricionista realizar consultas e diagnósticos nutricionais, bem como prescrição dietética, através da Internet ou qualquer outro meio de comunicação que configure atendimento não presencial". ${ }^{17}$

Após o cadastro, o usuário preenche seus dados pessoais de nome, gênero, data de nascimento, fator atividade física, peso, altura, e o aplicativo informa sobre sua classificação de IMC e necessidades diárias de energia e nutrientes (figura 2). 


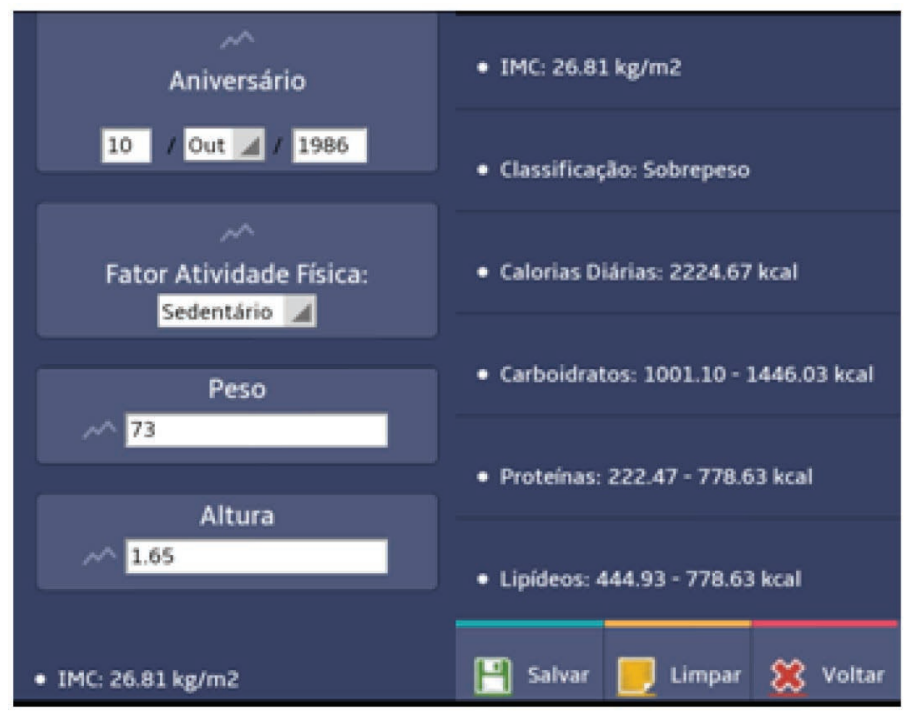

Figura 2. Tela "Perfil do usuário”. São Paulo, 2014.

O aplicativo Diet Help calcula as necessidades energéticas a partir das equações propostas pelas DRIs. Embora essa metodologia seja diferente da usada por outros pesquisadores como Lewandowski ${ }^{18}$ e Trindade et al., ${ }^{19}$ que utilizaram as fórmulas de Harris e Benedict, optou-se pelas equações das DRIs, por serem as mais adotadas recentemente.

A figura 3 ilustra a tela "Registro Alimentar". O registro alimentar (chamado de cadastro da dieta para melhor entendimento do usuário) é uma ferramenta que permite avaliar a dieta atual do indivíduo e estimar seu consumo energético e de nutrientes. Para isso, a pessoa deve anotar todos os alimentos e bebidas consumidos, ao longo de um ou mais dias. ${ }^{19}$ 


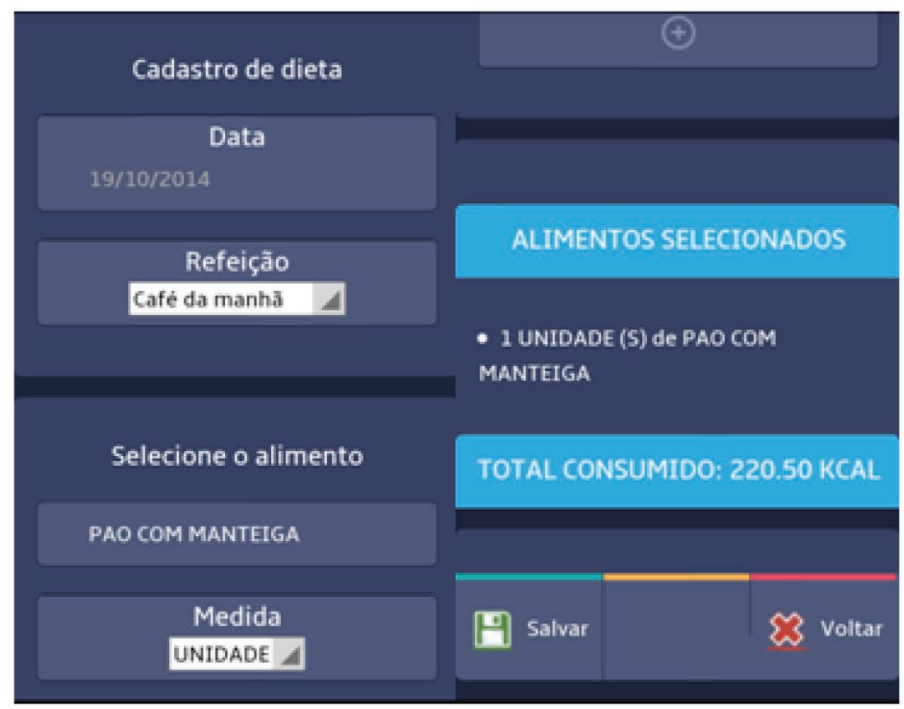

Figura 3. Tela “Registro Alimentar”. São Paulo, 2014.

O "Resumo do dia”, demonstrado na figura 4, permite ao usuário visualizar quantas calorias e nutrientes foram consumidos até o momento, de acordo com cada refeição. O aplicativo também informa se os valores estão de acordo ou inadequados segundo as recomendações.

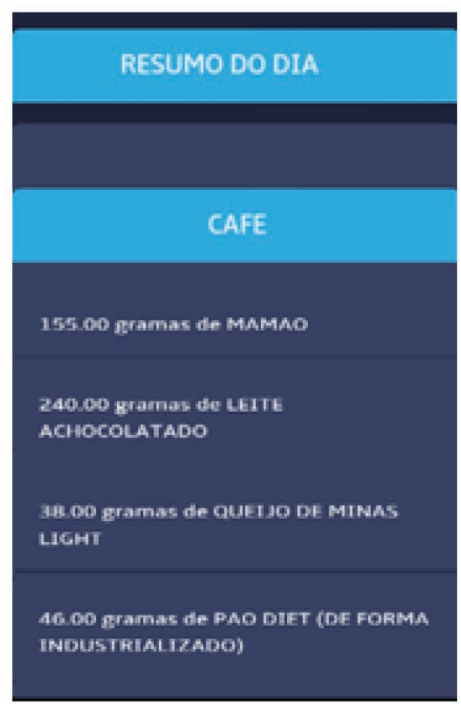

Figura 4. Tela "Resumo do dia”. São Paulo, 2014. 
O "Histórico da Dieta" (figura 5) é uma compilação do "Resumo do dia”. É possível ter acesso a todas as dietas cadastradas pelos usuários de acordo com os dias da semana. Com os dados de três, cinco ou sete dias, preferencialmente alternados, incluindo um dia do final de semana, é possível identificar as características do consumo atual, como os tipos de alimentos e preparações, assim como os horários mais frequentes das refeições. ${ }^{20}$

Na tela "Consulta aos alimentos", o usuário pode realizar a consulta da quantidade de calorias, macronutrientes e micronutrientes por $100 \mathrm{~g}$ de qualquer alimento que conste na Tabela do IBGE, conforme a figura 6 . O trabalho de Fernandes et al..$^{16}$ também permite ao usuário a consulta a alimentos e suas propriedades nutricionais - neste caso, o cadastro de alimentos para esta consulta é feito manualmente por um nutricionista.

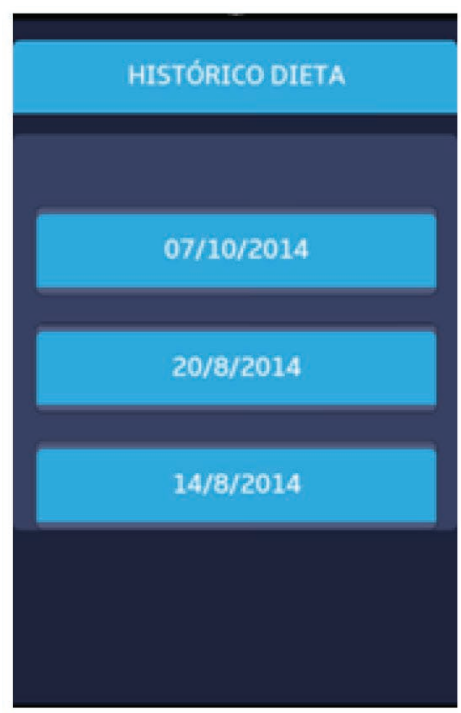

Figura 5. Tela "Histórico da dieta". São Paulo, 2014. 


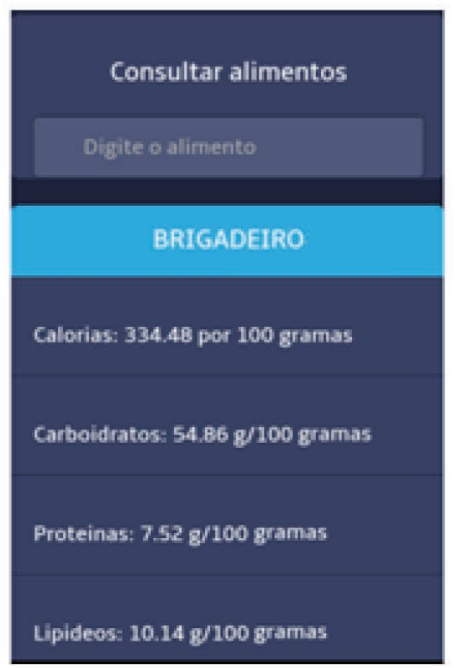

Figura 6. Tela "Consultar alimentos”. São Paulo, 2014.

A opção "Alimentação Saudável é composta por orientações baseadas nas recomendações propostas no Guia Alimentar para a População Brasileira ${ }^{15}$. O Guia Alimentar apresenta diretrizes para uma alimentação saudável com mensagens específicas para a população, podendo ser utilizado também por profissionais de saúde. ${ }^{21}$ Por fim, o usuário tem a opção de sair do aplicativo e excluir todas as informações cadastradas no Diet Help.

Assim, o aplicativo desenvolvido pretende ser mais uma ferramenta de orientação nutricional para auxiliar os usuários a escolher os alimentos de forma adequada e auxiliar na prevenção do desenvolvimento de doenças e melhoria da qualidade de vida.

\section{Conclusão}

Foi elaborado com sucesso um aplicativo de avaliação nutricional para telefones celulares com sistema Android. Essa ferramenta pode contribuir para que os indivíduos aprendam a fazer escolhas alimentares adequadas e prevenir o desenvolvimento de DCNTs.

O aplicativo desenvolvido foi nomeado Diet Help e utilizou as linguagens de programação Android Java, Apache Cordova, JavaScript, JQuery Mobile, HTML, CSS e SQLite. Oferece sete opções de funcionalidade ao usuário: Perfil do usuário, Registro alimentar, Resumo do dia, Histórico da dieta, Consultar alimentos, Alimentação Saudável e Excluir usuário. 


\section{Referências}

1. Magalhães JP, Souza RS, Souza JE, Hall- Nielsen RF, Alves JR, Faria LM, et al. Incentivo à adoção de modos saudáveis de vida entre profissionais de saúde: relato de uma intervenção na atenção primária. Rev. APS 2013; 16(2):202-206.

2. World Health Organization. Diet, nutrition and the prevention of chronic disease. Geneva: WHO; 2003. 150 p.

3. Ravasco P, Ferreira C, Camilo ME. Alimentação para a saúde: a relevância da intervenção dos médicos. Acta Med. Port. 2011; 24(4):783-790.

4. Ferreira SRG. Alimentação, nutrição e saúde: avanços e conflitos da modernidade. Cien. Cult. 2010; 63(4):31-34.

5. Proença RPC. Alimentação e globalização: algumas reflexões. Cien. Cult. 2010; 62(4):43-47.

6. Bezerra IN, Sichieri R. Características e gastos com alimentação fora do domicílio no Brasil. Rev. Saúde Pública 2010; 44(2):221-229.

7. Cruz DL, Paulo RRD, Dias WS, Martins VF, Gandolfi PE. O uso das mídias digitais na educação em saúde. Cadernos da FUCAMP 2011; 10(13):106-129.

8. Silva RV, Silva FA. Utilização de dispositivos móveis com acelerômetro para controle de aplicações. Colloquium Exactarum 2010; 2(1):12-20.

9. Batista SCF, Barcelos GT. Análise do uso do celular no educacional. CINTED-UFRGS 2013; 11(1):2-10.

10. Brasil. Ministério da Saúde. Curvas de Crescimento. Brasília: Ministério da Saúde; 2007. Disponível em: http://dab.saude.gov.br/portaldab/ape_vigilancia_alimentar.php?conteudo=curvas_de_crescimento

11. World Health Organization. BMI Classification. Genova: WHO; 2002. Disponível em: http://apps. who.int/bmi/index.jsp?introPage=intro_3.html

12. Organização Pan-Americana da Saúde. Encuestra multicêntrica: Salud Beinestar y Envejecimeiento (SABE) en América Latina e el Caribe. Informe preliminar. In: XXXVI Reunión del Comitê Asesor de Ivestigaciones en Salud; 9-11 jul. 2001; Kingston, Jamaica. Washington, D.C.: OPAS; 2001. Disponível em: http://envejecimiento.csic.es/documentos/documentos/paho-salud-01.pdf

13. Health Canada. Dietary reference intakes and recommennded dietary allowances. Takes and reports; 2004. Disponível em: http://www.hc-sc.gc.ca/fn-an/alt_formats/hpfb-dgpsa/pdf/nutrition/ dri_tables-eng.pdf

14. Instituto Brasileiro de Geografia e Estatísticas. Pesquisa de orçamentos familiares 2008-2009. Tabelas de composição nutricional dos alimentos consumidos no Brasil [Internet]. Disponível em: http://www. ibge.gov.br/home/estatistica/populacao/condicaodevida/pof/2008_2009_composicao_nutricional

15. Brasil. Ministério da Saúde. Guia alimentar para a população brasileira: promovendo a alimentação saudável. Brasília: Ministério da Saúde; 2008. 210 p. 
16. Fernandes FG, Oliveira LC, Barbosa AJ, Moura CCO, Oliveira FS. Sistema nutricional web e mobile - Nutrilife. In: XI CEEL. Universidade Federal de Uberlândia, 25-29 nov. 2013. Uberlândia: UFU; 2013. Disponível em: http://www.ceel.eletrica.ufu.br/artigos2013/ceel2013_026.pdf

17. Conselho Federal de Nutricionistas (Brasil). Código de ética do nutricionista. Resolução CFN n 334/2004. Brasília: CFN; 2004. Disponível em: http://www.cfn.org.br/novosite/pdf/codigo/ codigo $\% 20$ de $\% 20$ etica_nova $\% 20$ redacao.pdf

18. Lewandowski A. Aplicativo móvel para controle diários de calorias ingeridas e gastas por uma pessoa [dissertação]. Curitiba: Universidade Tecnológica Federal do Paraná; 2011.

19. Trindade F, Mantau MJ, Berkenbrock CDM. Desenvolvimento e avaliação de uma ferramenta móvel para avaliação nutricional. Anais SULCOMP 2012; 6(1):50-55.

20. Fisberg RM, Marchioni DML, Colucci ACA. Avaliação do consumo alimentar e da ingestão de nutrientes na prática clínica. Arq. Bras. Endocrinol. Metab. 2009; 53(5):617-624.

21. Jaime PC, Silva ACF, Lima AMC, Bortolini GA. Ações de alimentação e nutrição na atenção básica: a experiência de organização no Governo Brasileiro. Rev. Nutr. 2011; 24(6):809-824.

Recebido: $13 / 3 / 2015$

Revisado: $21 / 9 / 2015$

Aprovado: 18/10/2015 